\title{
Epiphysial Separation of Femoral Head Due to Undiagnosed Hip Septic Arthritis
}

\author{
Mohammad Hosein Ebrahimzadeh ${ }^{1}$; Farshid Bagheri ${ }^{1}$; Masoud Mirkazemi ${ }^{1}$; Mehran \\ Azami $^{1}$; Ali Birjandinejad ${ }^{1,2,}$ \\ ${ }^{1}$ Orthopedic Research Center, Ghaem Hospital, Mashhad University of Medical Sciences, Mashhad, IR Iran \\ 2 Orthopedic Research Center, Shahid Kamyab Hospital, Mashhad University of Medical Sciences, Mashhad, IR Iran \\ ${ }^{*}$ Corresponding author: Ali Birjandinejad, Orthopedic Research Center, Shahid Kamyab Hospital, Mashhad University of Medical Sciences, Mashhad, IR Iran. Tel: +98-5118518853, Fax: \\ +98-5118549234, E-mail: Birjandinejada@mums.ac.ir
}

Received: January 20, 2014; Revised: February 27, 2014; Accepted: April 12, 2014

\begin{abstract}
Introduction: Slipped capital femoral epiphysis (SCFE) is one of the complications of septic arthritis that occurs rarely in children after septic arthritis of the hip. Septic arthritis is uncommon in neonates and therefore proper assessment and treatment may be problematic. Early treatment of septic arthritis could prevent SCFE.

Case Presentation: This complication happened in our patient who was in fact a neglected child due to septic arthritis of the hip and femoral head epiphysis, neck osteomyelitis and delayed treatment. At the time of admission and hospitalization, femoral head was palpable at the lateral side of the middle of thigh and a fistula had been created in that spot.

Conclusions: This phenomenon has been reported as a rare complication of septic arthritis and osteomyelitis. Treatment of these patients is controversial and the long term results are unknown but efforts must be done for early diagnosis and anatomic reduction of femoral epiphysis.
\end{abstract}

Keywords:Femur Head; Separation; Hip; Arthritis, Infectious; Delayed Diagnosis; Osteomyelitis

\section{Introduction}

Hematogenous osteomyelitis and septic arthritis in children can occur simultaneously. Approximately 20\% of children (children under seven years old) with septic arthritis have adjacent osteomyelitis but about 50\% of infants with infectious arthritis are associated with osteomyelitis $(1,2)$. Epiphysial plate in older children acts as a barrier to prevent entrance of infection to the joint space but in children under seven years old, arteries pass through the physis and epiphysial plate and enter the joint space as a result, this can cause septic arthritis and other complications. Some of these complications are hip dislocation, proximal femoral physial arrest (that may lead to limb shortening), femoral head avascular necrosis (AVN), Coxa magna and Coxa vara and also early osteoarthritis (3-5).

Septic arthritis and associated osteomyelitis are uncommon in developed countries. Most cases of SCFE have been reported in children under seven years old and the majority of these reports refer to the time when emergent hip arthrotomy was not performed as a standard treatment in hip infectious arthritis $(6,7)$. Diagnosis and treatment of Physial separation in infants and children need special attention. The treatment must be done as soon as possible (8).

\section{Case Presentation}

The patient was a 7-year-old boy who was mental retarded with complaint of right thigh pain and inability to walk since 2.5 months before admission. An ulcer was gradually developed and a fistula was formed in the lateral side of the right thigh in the size of $1 \mathrm{~cm}$. Two weeks after appearance of fistula, the patient was referred for treatment. Since this case was a neglected child, no kinds of treatment had been done before he was referred to our clinic with fistula. In his medical history, he was able to walk independently but not as normal gait before the appearance of the symptoms. After his admission in operating room, culture samples were taken from the fistula discharge through which Staphylococcus aureus was detected, so the antibiotic therapy began. In radiologic assessment, epiphyseal separation of femoral head was observed in his radiographs (Figures 1 and 2). Blue methylen was injected through the fistula and one day after injection, debridement and removal of the femoral head through the fistula were performed, the fistula path was removed and hip arthrotomy was done, and the culture from hip joint was taken in which Staphylococcus aureus again was detected. After that, treatment continued with skin traction and antibiotic therapy. One week after 


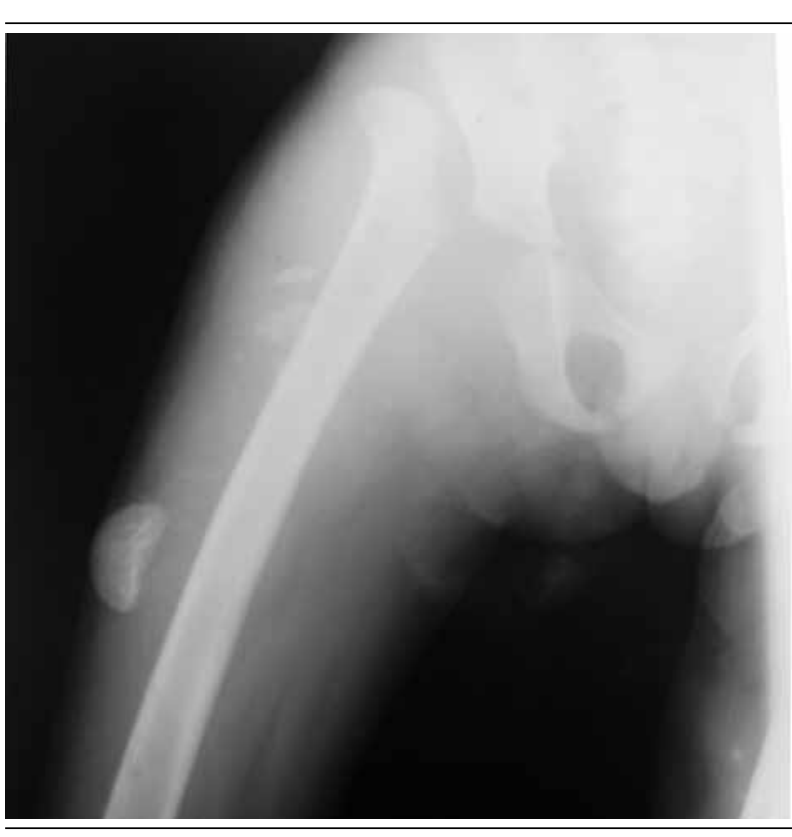

Figure 1. Epiphysial Separation of Right Femoral Head and Migration of Femoral Head Epiphysis to Lateral Side of the Thigh and Hip Dislocation and Proximal Migration of Femur

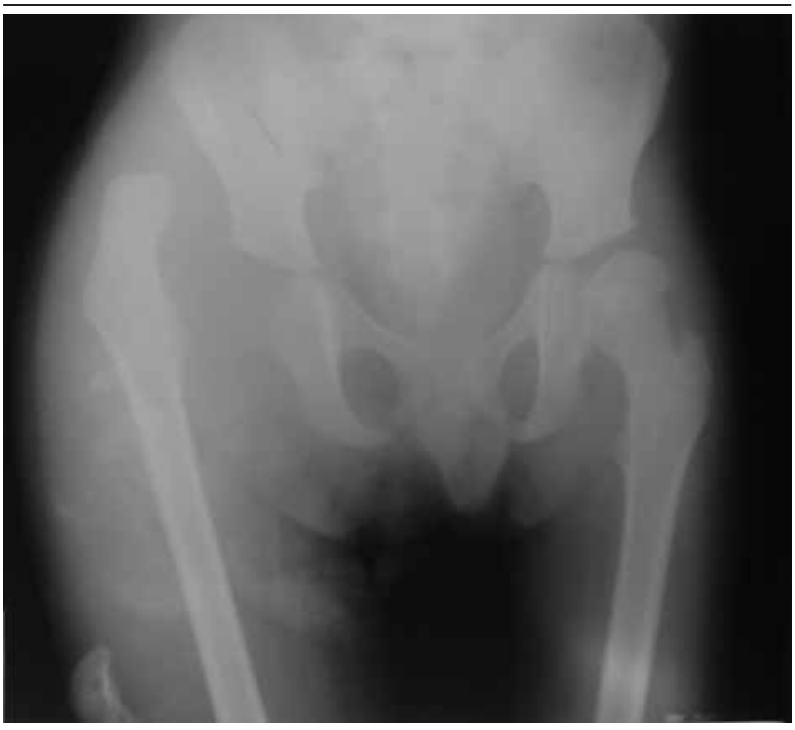

Figure 2. Ap View of the Pelvis Radiography Shows Proximal Dislocation of Femoral Head and Epiphysial Separation of Femoral Head

application of traction, the remnant of femoral head was placed in the acetabulum and immobilized with spica cast.

After 6 weeks, the cast was removed and range of motion began. After 6 months, overall health was very good and there were no abnormal clinical signs and laboratory findings (Erythrocyte Sedimentation Rate, ESR, was normal and C-reactive protein, CRP, was Negative). Range of motion of the hip was $90^{\circ}$ flexion, $50^{\circ}$ abduction, and $20^{\circ}$ adduction and internal and external rotation was within acceptable limits. The patient was able to walk without assistance.

\section{Discussion}

Although epiphysial separation is rare in infants and children, the most common site of involvement is femoral head $(9,10)$. Complete detachment of femoral head epiphysis is extremely rare in cases with hip septic arthritis. It has been reported in several studies that the hip joint is the most common site of slipped epiphysial as well as the most common site for septic arthritis in infants $(11,12)$. Because of anatomical and histological characteristics of the proximal femur (large sinusoidal vessels, cancellous metaphysis of femoral head, thin cortex, and incomplete periosteal attachment) in children, infection in the epiphysial area can easily spread to the subperiosteal area, the joint and soft tissue levels. Bone destruction near the epiphysial area associated with periosteal detachment leads to loosening of femoral head epiphyseal. This phenomenon can cause two complications including septic arthritis and epiphysial separation that are only seen in neonates and children (13).

In our patient, there were some risk factors for the development of septic arthritis including mental retardation and lack of parental care. The history of trauma in this patient was not clear. There was no evidence of metabolic disease and child abuse. Early diagnosis of epiphysial separation after trauma or septic arthritis is difficult. Clinical signs of septic arthritis and osteomyelitis are usually observed but more laboratory and clinical assessment is needed $(14,15)$. Our patient had no signs of hip septic arthritis. Samples were taken from the purulent discharge from the fistula for smear and culture. For confirmation, diagnostic samples were taken again after the debridement for smear and culture in which Staphylococcus aureus infection was reported. It should be noted that in standard pelvis radiography of infants, the femoral head epiphysis is not seen due to lack of ossification. As the child grows, the central core of femoral head epiphysis gradually appears. For diagnosis, standard radiographs of the pelvis and proximal femur should be performed that requires the proper position of the patient; but in many cases it could not clearly be distinguished due to lack of cooperation from infants and their parents. Ultrasound can also be used to detect fluid in the hip joint (16). Hip and pelvis radiographies were done for our patient and the epiphysial femoral head was evident in lateral side of the femur. After these procedures the patient was diagnosed as epiphysial separation of femoral head due to undiagnosed septic arthritis. Partial or complete detachment of the femoral head has been reported as a complication of the hip septic arthritis and proximal femoral osteomyelitis (17). However, based on our findings, complete detachment and migration of femoral head to the lateral side of femur have not been reported yet. In this patient, due to complete destruction of metaphyseal portion of the femoral head and osteomyelitis of femoral epiphysis, reduction was impossible. Thus, the epiphysial segment was removed and after debridement 
of the infected tissues, the remaining proximal femoral epiphysis was placed in the acetabular cavity and spica cast was applied.

Early diagnosis and proper treatment of these patients will lead to good results. Reduction and pin fixation have been reported as a treatment for epiphysial femoral head separation $(18,19)$. Despite the rare case of association of osteomyelitis with epiphysial separation of femoral head in infants and children, this diagnosis should be considered by physicians for infants and children. Diagnosis, especially in children and patients with mental retardation can be somewhat difficult. According to various published reports, these patients are often missed or diagnosed late, so the treatment results would be undesirable.

\section{Acknowledgements}

The authors are thankful to Nahid Mojaver for making communication between authors and help to translate.

\section{Author's Contribution}

Case study: Dr. Ali Birjandinejad; interpretation of data: Dr. Masoud Mirkazemi; draft: Dr. Mohammad Hosein Ebrahimzadeh and Dr. Farshid Bagheri; operations and follow up: Dr. Ali Birjandinejad and Dr. Mehran Azami. All authors finally read the paper and approved its content.

\section{Financial Disclosure}

There is no conflict of interest.

\section{Funding/Support}

There is no financial interest.

\section{References}

1. Petersen S, Knudsen FU, Andersen EA, Egeblad M. Acute haematogenous osteomyelitis and septic arthritis in childhood. A 10-year review and follow-up. Acta Orthop Scand.1980;51(3):451-7.
2. Jackson MA, Burry VF, Olson LC. Pyogenic arthritis associated with adjacent osteomyelitis: identification of the sequelaprone child. Pediatr Infect Dis J.1992;11(1):9-13.

3. Daniel D, Akeson W, Amiel D, Ryder M, Boyer J. Lavage of septic joints in rabbits: effects of chondrolysis. J Bone Joint Surg Am. 1976;58(3):393-5.

4. Gillespie R. Septic arthritis of childhood. Clin Orthop Relat Res. 1973(96):152-9.

5. Fabry G, Meire E. Septic arthritis of the hip in children: poor results after late and inadequate treatment. J Pediatr Orthop. 1983;3(4):461-6.

6. Morrey BF, Bianco AJ, Rhodes KH. Suppurative arthritis of the hip in children. J Bone Joint Surg Am. 1976;58(3):388-92.

7. Paterson DC. Acute suppurative arthritis in infancy and childhood. J Bone Joint Surg Br. 1970;52(3):474-82.

8. Badgley CE, Yglesias L, Perham WS. Study of the end results in 113 cases of septic hips. J Bone Joint Surg Am. 1936;18(4):1047-61.

9. Dessi A, Crisafulli M, Accossu S, Setzu V, Fanos V. Osteo-articular infections in newborns: diagnosis and treatment. J Chemother. 2008;20(5):542-50.

10. Nade S. Acute haematogenous osteomyelitis in infancy and childhood. J Bone Joint Surg Br. 1983;65(2):109-19.

11. Sferopoulos NK, Papavasiliou VA. [Proximal epiphyseal separation of the femur in the newborn: early ultrasonic diagnosis]. Rev Chir Orthop Reparatrice Appar Mot. 1994;80(4):338-41.

12. Steer AC, Carapetis JR. Acute Hematogenous Osteomyelitis in Children. Pediatric Drugs. 2004;6(6):333-46.

13. Ogden JA, Lister G. The pathology of neonatal osteomyelitis. Pediatrics. 1975;55(4):474-8.

14. Okubo T, Yabe S, Otsuka T, Takizawa Y, Takano T, Dohmae S, et al. Multifocal pelvic abscesses and osteomyelitis from community-acquired methicillin-resistant Staphylococcus aureus in a 17-year-old basketball player. Diagn Microbiol Infect Dis. 2008;60(3):313-8.

15. Crary SE, Buchanan GR, Drake CE, Journeycake JM. Venous thrombosis and thromboembolism in children with osteomyelitis. J Pediatr. 2006;149(4):537-41.

16. Aroojis AJ, Johari AN. Epiphyseal separations after neonatal osteomyelitis and septic arthritis. J Pediatr Orthop. 2000;20(4):544-9.

17. Graf R. Sonographie der Sauglingshufte.Stuttgart: Ferdinand Enkle Verlag; 1986.

18. Ogden JA. Pediatric osteomyelitis and septic arthritis: the pathology of neonatal disease. Yale J Biol Med.1979;52(5):423-48.

19. Mangurten HH, Puppala B, Knuth A. Neonatal distal femora physeal fracture requiring closed reduction and pinning.J Perinatol. 2005;25(3):216-9. 